\title{
Update on CAR T-Cell Therapies for Relapsed/Refractory B-Cell Lymphomas
}

\author{
Presented by Stephen J. Schuster, MD
}

\begin{abstract}
CAR T cells are genetically engineered to produce an artificial receptor on the T-cell surface for use in immunotherapy, which immediately engages its target, results in CAR T-cell proliferation, and effects target cell cytotoxicity. Prior to CAR T-cell therapy, approximately $30 \%$ of patients with diffuse large B-cell lymphoma (DLBCL) had an unmet need for a new treatment approach. There are now 3 commercially available CAR T-cell products capable of achieving long-term, disease-free survival in the third line of therapy for patients with relapsed or refractory DLBCL, transformed follicular lymphoma, or primary mediastinal large B-cell lymphoma: axicabtagene ciloleucel, tisagenlecleucel, and lisocabtagene maraleucel.
\end{abstract}

J Natl Compr Canc Netw 2021;19(5.5):622-624 doi: $10.6004 /$ jnccn.2021.5017

Over the past 25 years, immunotherapy-based approaches to cancer treatment have become a standard of care for various tumor types, and immunotherapy is now considered the "fourth leg" of clinical oncology. During the NCCN 2021 Virtual Annual Conference, Stephen J. Schuster, MD, Professor of Medicine, Perelman School of Medicine at the University of Pennsylvania, and Director, Lymphoma Program and Lymphoma Translational Research, Abramson Cancer Center of the University of Pennsylvania, discussed CD19-directed CAR T-cell therapies for relapsed/refractory B-cell lymphomas. "Given the unprecedented bench-to-bedside clinical success, research and development aimed at refining our existing immunotherapies is proceeding at a very rapid pace," said Dr. Schuster. "It's a very exciting time for oncologists."

\section{CAR T-Cell Therapy}

Unlike the normal immune response, CAR T cells, which are engineered by gene therapy to have a chimeric receptor, do not require MHC-restricted antigen presentation. As Dr. Schuster explained, CAR T-cell therapy is a very direct approach. The chimeric receptor has a single-chain variable fragment derived from an antibody for binding a tumor antigen, which binds with very high affinitymuch higher than an MHC-bound peptide binds to a $\mathrm{T}$-cell receptor. It also has a costimulatory module in the intracellular domain of the chimeric receptor, which is the "on switch" for proliferation and cytotoxicity. "These cells are engineered to immediately engage targets, proliferate, and effect cytotoxicity," he said.

Prior to the advent of CAR T-cell therapy, first-line treatment of diffuse large B-cell lymphoma (DLBCL) was rituximab + chemotherapy; 5-year overall survival is approximately 55\% with this approach. Second-line treatment with high-dose chemotherapy + autologous stem cell transplant (SCT) would cure an additional $14 \%$ of patients with DLBCL, including those who fail to respond to primary rituximab + chemotherapy. According to Dr. Schuster, this left approximately $30 \%$ of patients with DLBCL with an unmet need in the pre-CAR T-cell era, which was the impetus for the development of this technology.

\section{CD19-Directed CAR T Cells}

Currently, there are 3 different CD19-directed CAR T-cell products approved for use in relapsed/refractory large B-cell lymphomas: axicabtagene ciloleucel (axi-cel), approved in late 2017; tisagenlecleucel (tisa-cel), approved in early 2018; and lisocabtagene maraleucel (liso-cel), approved this year (Table 1). ${ }^{1-3}$ All 3 are CD19-directed, but tisa-cel and liso-cel use a 4-1BB costimulatory domain, and axi-cel uses a CD28 costimulatory domain. This affects the rate of expansion and the pharmacokinetics and, to some extent, mediates toxicities, said Dr. Schuster. All 3 CAR T-cell products are approved for relapsed/ refractory DLBCL in the third-line setting, where patients have either received second-line SCT and then experienced a recurrence or were unable to receive SCT due to disease refractory to second-line therapy.

\section{Axi-Cel}

The ZUMA-1 trial enrolled patients with relapsed or refractory large B-cell lymphomas into 2 cohorts: DLBCL or high-grade B-cell lymphoma (HGBCL) and transformed follicular lymphoma (FL) or primary mediastinal B-cell lymphoma. Two-thirds of patients were refractory to the 


\begin{tabular}{|c|c|c|c|}
\hline & Axicabtagene Ciloleucel ${ }^{1}$ & Tisagenlecleucel $^{2}$ & 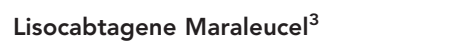 \\
\hline Construct & antiCD19-CD28-CD3 $\zeta$ & antiCD19-41BB-CD3 $\zeta$ & antiCD19-41BB-CD3 $\zeta$ (tEGFR) \\
\hline Apheresis product & Bulk, fresh PBMC & Bulk, cryopreserved PBMC & Fresh, isolated $\mathrm{CD} 8+$ and $\mathrm{CD} 4+\mathrm{T}$ cells \\
\hline CAR + cell dose & $2 \times 10^{6} / \mathrm{kg}\left(\max 2 \times 10^{8}\right)$ & 0.6 to $6.0 \times 10^{8}$ & $100 \times 10^{6} \mathrm{CD} 8+\& \mathrm{CD} 4+$ cells \\
\hline Bridging therapy & $\begin{array}{l}\text { None in pivotal trial, often used } \\
\text { in standard practice }\end{array}$ & $92 \%$ of patients in pivotal trial & $59 \%$ of patients in pivotal trial \\
\hline Lymphodepletion & Cy/Flu, $500 / 30 \times 3 d$ & $\begin{array}{l}\mathrm{Cy} / \mathrm{Flu}, 250 / 25 \times 3 \mathrm{~d} \text {, or } \\
\text { bendamustine } 90 \times 2 \mathrm{~d}\end{array}$ & Cy/Flu, $300 / 30 \times 3 d$ \\
\hline Approval status & FDA/EMA-approved & FDA/EMA-approved & FDA-approved \\
\hline
\end{tabular}

Abbreviations: Cy/Flu, cyclophosphamide/fludarabine; EMA, European Medicines Agency; max, maximum; PBMC, peripheral blood mononuclear cells.

antecedent therapy. ${ }^{1}$ Study results demonstrated that approximately $40 \%$ of patients were alive and progressionfree at 24 months versus the expected $4 \%$ of those receiving third-line treatment in the pre-CAR T-cell era.

"Interestingly, most failures occur within the first 3 to 5 months," said Dr. Schuster. "The progression-free survival [PFS] and response duration curves begin to plateau between 3 and 6 months, and there are very few events that occur after 1 year."

\section{Tisa-Cel}

The JULIET trial, ${ }^{2}$ a phase II global trial of tisa-cel, enrolled patients with DLBCL or transformed FL, and more than half of the patients were refractory to their antecedent therapy. Overall response rates and complete response rates were $54 \%$ and $40 \%$, respectively. According to Dr. Schuster, however, response rates don't tell the whole story.

"What really matters, in my opinion, is whether the patient is maintaining a response at 3, 6, and 12 months," he said, reporting that approximately one-third of patients receiving tisa-cel are disease-free at 1 year. "That's how you should judge the products." Furthermore, he explained, patients in remission at 3 months have approximately a $70 \%$ chance of long-term disease-free survival (DFS), and those in complete remission at 12 months have a $\geq 95 \%$ chance of long-term DFS.

\section{Lisa-Cel}

The Transcend NHL001 trial of liso-cel included patients with DLBCL; HGBCL with rearrangements of MYC and either BCL2, BCL6, or both (double-hit or triple-hit lymphomas); DLBCL transformed from any indolent lymphoma; primary mediastinal B-cell lymphoma (PMBCL); and FL grade 3B. ${ }^{3}$ Approximately two-thirds of patients were chemotherapy-refractory and nearly half had never achieved a complete response with any antecedent therapies. At 12 months, PFS for all patients was $44 \%$. Patients with a complete response $(53 \%)$ had a 12 -month PFS of 65\%. "With this study, we see a similar pattern of early failures followed by a plateau, which begins between 3 and 6 months," said Dr. Schuster. "After 1 year, there were very few events."

Because each of the trials differed with respect to the composition of patient histology, Dr. Schuster emphasized that the outcomes between each study cannot be compared. However, he noted that patients with HGBCL and DLBCL performed worse than those with PMBCL and transformed FL.

"The takeaway message is that we have 3 active products that all are capable of achieving long-term DFS in the third line of therapy for patients with DLBCL, transformed FL, or PMBCL," said Dr. Schuster. "There are pros and cons in terms of logistics, but these represent the best options for patients in the third-line setting" (Table 2).

\section{CAR T-Cell-Related Toxicities}

There are some differences in toxicities seen between the available CAR T-cell products. Cytokine release syndrome (CRS) is seen in all studies, but real-world data indicate that grade 3 CRS is in the single digits, said Dr. Schuster, who noted that clinicians have "learned to mitigate this syndrome" by using tocilizumab earlier.

Although neurotoxicity is seen across the board with all 3 products, the products with 4-1BB costimulatory domains (tisa-cel and liso-cel) have a lower frequency of neurotoxicity and CRS events than the CD28 costimulated products, Dr. Schuster reported. ${ }^{4,5}$

The NCCN Clinical Practice Guidelines in Oncology (NCCN Guidelines) for Management of ImmunotherapyRelated Toxicities provide guidance for management of CRS and neurotoxicity. Tocilizumab, an anti-IL-6 antibody, is the cornerstone of treatment for CRS. In most patients, tocilizumab can stop CRS immediately, said Dr. Schuster. Neurotoxicity is generally treated with steroids and is frequently reversible.

\section{Approaches for Improving Outcomes}

Despite the success of CAR T-cell therapy, Dr. Schuster emphasized that there is still a large unmet need for 
Table 2. CD19 CAR T-Cell Therapies for DLBCL: Summary

\begin{tabular}{|c|c|c|c|c|c|c|c|c|c|}
\hline \multirow[b]{2}{*}{ Disease state } & \multicolumn{2}{|c|}{ Tisagenlecleucel $^{2}$} & \multicolumn{2}{|c|}{ Axicabtagene Ciloleucel ${ }^{1,4}$} & \multicolumn{5}{|c|}{${\text { Lisocabtagene } \text { Maraleucel }^{3}}^{3}$} \\
\hline & $\mathrm{r} / \mathrm{r} \mathrm{DLBCL}$ & $\mathrm{r} / \mathrm{rtFL}$ & $\mathrm{r} / \mathrm{r} \mathrm{DLBCL}$ & $\mathrm{r} / \mathrm{r} \mathrm{tFL} / \mathrm{PMBCL}$ & $\mathrm{r} / \mathrm{r} \mathrm{DLBCL}$ & $\mathrm{t}$ iNHL & PMBCL & $\mathrm{HGBCL}$ & FL3B \\
\hline Response evaluable, $n$ & 89 & 22 & 77 & 24 & 137 & 78 & 15 & 36 & 3 \\
\hline Follow-up, median & \multicolumn{2}{|c|}{$14 \mathrm{mo}$} & \multicolumn{2}{|c|}{$15.4 \mathrm{mo}$} & \multicolumn{5}{|c|}{$12.3 \mathrm{mo}$} \\
\hline Efficacy & \multicolumn{2}{|c|}{$\mathrm{n}=93$} & \multicolumn{2}{|c|}{$\mathrm{n}=101$} & \multicolumn{5}{|c|}{$\mathrm{n}=256$} \\
\hline ORR/CR & \multicolumn{2}{|c|}{$52 \% / 40 \%$ [best] } & \multicolumn{2}{|c|}{$82 \% / 54 \%$ [best] } & \multicolumn{5}{|c|}{ 73\%/53\% [best] } \\
\hline$\%$ PFS for CR at $12 \mathrm{mo}$ & \multicolumn{2}{|c|}{$78.50 \%$} & \multicolumn{2}{|c|}{$79 \%$} & \multicolumn{5}{|c|}{$65 \%$} \\
\hline DoR (CR/PR; median) & \multicolumn{2}{|c|}{ NR } & \multicolumn{2}{|c|}{$\begin{array}{l}11.1 \text { months } \\
\text { (NR by IRC) }\end{array}$} & \multicolumn{5}{|c|}{$\begin{array}{c}\text { NR for PMBCL \& tFL } \\
\text { DLBCL: } 5.6 \text { mo; HGBCL: } 10.8 \mathrm{mo}\end{array}$} \\
\hline DoR (CR; median) & \multicolumn{2}{|c|}{ NR } & \multicolumn{2}{|c|}{ NR } & \multicolumn{5}{|c|}{ NR } \\
\hline Safety & \multicolumn{2}{|c|}{$\mathrm{n}=111$} & \multicolumn{2}{|c|}{$\mathrm{n}=101$} & \multicolumn{5}{|c|}{$\mathrm{n}=269$} \\
\hline CRS & \multicolumn{2}{|c|}{$22 \%$ grade $3 / 4^{a}$} & \multicolumn{2}{|c|}{$13 \%$ grade $\geq 3^{b}$} & \multicolumn{5}{|c|}{$2 \%$ grade $3 / 4^{b}$} \\
\hline Neurotoxicity & \multicolumn{2}{|c|}{$12 \%$ grade $3 / 4$} & \multicolumn{2}{|c|}{$28 \%$ grade $\geq 3$} & \multicolumn{5}{|c|}{$10 \%$ grade $3 / 4$} \\
\hline
\end{tabular}

Abbreviations: $C R$, complete response; $C R S$, cytokine release syndrome; DLBCL, diffuse large B-cell lymphoma; DoR, duration of response; $H G B C L$, highgrade B-cell lymphoma; NR, not reached; ORR, overall response rate; PFS, progression-free survival; PMBCL, primary mediastinal B-cell lymphoma; PR, partial response; $r / r$, relapsed/refractory; $\mathrm{iNHL}$, transformed indolent non-Hodgkin lymphoma; $\mathrm{tFL}$, transformed follicular lymphoma.

aPenn scale.

${ }^{\mathrm{b}}$ Lee scale.

patients. "We're curing 3 times as many patients as we used to in the third-line setting, but only a third of these third-line patients with aggressive B-cell lymphomas are successfully treated, and there's still a significant unmet need for many of these patients," he said. "Approximately half of the patients who fail first-line chemotherapy never make it to autologous SCT. Although so these patients could potentially receive CAR T-cell therapy." One way to improve outcomes is better selection of patients. Another key is to achieve disease stability prior to CAR T therapy, because patients whose disease is progressing tend to not respond well to therapy. "You need to at least achieve disease stability," said Dr. Schuster. "Tumor bulk, serum lactate dehydrogenase, and performance status will also affect outcome."

"Apheresis and cell manufacturing are correlated with success or failure as well," he continued. "Success depends on how much prior therapy a patient has undergone and the phenotype of the pheresis product. The quality of the product will impact outcome."

Finally, bridging therapy is the "art of oncology," said Dr. Schuster, and there are new agents, such as polatuzumab vedotin and ibrutinib, that can help carry a patient to CAR T-cell treatment.

Disclosures: Dr. Schuster has disclosed receiving consulting fees from Allogene Therapeutics, AstraZeneca Pharmaceuticals LP, BeiGene, Celgene Corporation, Genentech, Inc., Incyte Corporation, Janssen Pharmaceutica Products, LP, Legend Biotech, Loxo Oncology, Onc., Novartis

Pharmaceuticals Corporation, and Regeneron Pharmaceuticals, Inc.; serving as a scientific advisor for Celgene Corporation, Genentech, Inc., Loxo Oncology, Onc., and Novartis Pharmaceuticals Corporation; receiving grant/research support from Genentech, Inc. and Novartis Pharmaceuticals Corporation; and receiving honoraria from Novartis Pharmaceuticals Corporation.

Correspondence: Stephen J. Schuster, MD, Abramson Cancer Center at the University of Pennsylvania, 3400 Civic Center Boulevard, PCAM, Floor 12, Room 12-178, Philadelphia, PA 19104

Email: schustes@pennmedicine.upenn.edu

\section{References}

1. Locke FL, Ghobadi A, Jacobson CA, et al. Long-term safety and activity of axicabtagene ciloleucel in refractory large B-cell lymphoma (ZUMA-1): a single-arm, multicentre, phase 1-2 trial. Lancet Oncol 2019;20:31-42.

2. Schuster SJ, Bishop MR, Tam CS, et al. Tisagenlecleucel in adult relapsed or refractory diffuse large B-cell lymphoma. N Engl J Med 2019;380:45-56.

3. Abramson JS, Palomba ML, Gordon LI, et al. Lisocabtagene maraleucel for patients with relapsed or refractory large B-cell lymphomas
(TRANSCEND NHL 001): a multicentre seamless design study. Lancet 2020;396:839-852.

4. Neelapu SS, Locke FL, Bartlett NL, et al. Axicabtagene ciloleucel CAR T-cell therapy in refractory large B-cell lymphoma. N Engl J Med 2017;377:2531-2544.

5. Schuster SJ, Maziarz RT, Rusch ES, et al. Grading and management of cytokine release syndrome in patients treated with tisagenlecleucel in the JULIET trial. Blood Adv 2020;4:1432-1439. 